\title{
Surface Texturing with Hemispherical Cavities to Improve Efficiency in Silicon Solar Cells
}

\author{
D. W. de Lima Monteiro, ${ }^{1}$ F. P. Honorato, ${ }^{2}$ R. F. de Oliveira Costa, ${ }^{1}$ and L. P. Salles ${ }^{1}$ \\ ${ }^{1}$ OptMA Lab, Department of Electrical Engineering, Universidade Federal de Minas Gerais, Avenida Antônio Carlos, 6627, \\ 31270-010 Belo Horizonte, MG, Brazil \\ ${ }^{2}$ Secretaria Municipal de Obras e Infraestrutura, Prefeitura Municipal de Belo Horizonte, Avenida do Contorno, 5454, \\ 30110-100 Belo Horizonte, MG, Brazil \\ Correspondence should be addressed to D. W. de Lima Monteiro, davies@ufmg.br
}

Received 31 August 2011; Revised 3 November 2011; Accepted 14 November 2011

Academic Editor: Stephen Bremner

Copyright ( 2012 D. W. de Lima Monteiro et al. This is an open access article distributed under the Creative Commons Attribution License, which permits unrestricted use, distribution, and reproduction in any medium, provided the original work is properly cited.

\begin{abstract}
Improvement of solar-cell efficiency at a minimum possible cost addition is constantly sought, and this is often achieved at incremental percentage steps. Among a number of alternatives, antireflective coatings and surface texturing are the most prominent. This paper presents an alternative texturing method of crystalline silicon in an attempt to improve the efficiency of photon transmission through the surface and collection in the bulk. The method relies on anisotropic etching of bulk silicon and requires only a single oxide mask and two etching steps with a $\mathrm{KOH}$ or TMAH aqueous solution. The surface texture consists of smooth hemispherical cavities, which do not demand a lithographic mask or intricate technology processes to obtain the hemispherical cavities. This method can be applied to increase the profile area of the originally flat frontal surface exposed to light and consequently increase the effective width of the depletion region. The latter implies a higher probability of photon collection, contributing to the improvement of the conversion efficiency of the device. The textured nontilted silicon solar-cell transmittance under small solar incidence angles at dawn and sunset is improved compared to a flat surface, increasing the photocurrent.
\end{abstract}

\section{Introduction}

Very often texturing is employed to increase the solar-cell surface area $[1,2]$. In some methods, based on inverted pyramids or laser-induced cone-shaped pillars, internal optical confinement of long-wavelength photons can be enhanced by light trapping [3,4]. Other methods employ parasitic hillocks originating from sticking $\mathrm{H}_{2}$ bubbles and precipitates, especially in low-concentration etching solutions [5]. More recently, nanostructures and wires have been proposed $[6,7]$.

The overall efficiency of a solar cell also depends on several aspects, among which, the composition of the semiconductor, temperature, contacts and metallization, antireflection coatings (ARC) type, doping concentration and topology of the cell layers, and encapsulation. As is widely known, silicon is predominantly used for the manufacture of photovoltaic cells because it is abundant in nature, processing technologies are mature and the energy bandgap is compatible with most of the solar spectrum. Nowadays, the world production of solar cells is largely concentrated in multi/micro/polycrystalline (m-Si, $\mu$-Si, p-Si) and monocrystalline silicon (c-Si), with the latter featuring the highest efficiencies.

We present a method to texture the silicon surface to improve optical transmittance into the cell, especially at low sun-elevation angles. In this method a mesh of pyramidal pits in silicon gradually evolves into a mesh of hemispherical cavities by means of a single-step anisotropic etching with a $\mathrm{KOH}$ aqueous solution [8]. The method was initially proposed for the fabrication of micromirrors and arbitrarily shaped optical surfaces $[8,9]$. The cross-disciplinary concept for texturing originated from our previous experience with the method for the latter applications.

Several improvements can be experimented with this texturing technique. Doping after texturing, either by diffusion 
or by ion implantation, results in an emitter-base junction that mimics the surface profile, consequently enhancing the volume of the depletion layer and yields a higher probability of photogenerated carrier collection, especially by drift. Alternatively, the textured junction can also be achieved by depositing a doped emitter thin film on the textured substrate. Moreover, the spherical depressions can serve as high-refraction index concave lenses to enhance long-wavelength absorption. Also, the textured surface serves as perfect molding for polymeric or low-temperature glass microlens-array solar concentrators [9].

In this paper we focus primarily on this alternative texturing idea, and present a preliminary performance analysis based on the influence of the proposed texturing on the transmittance at the air-silicon interface, representing the portion of the solar radiation effectively penetrating the cell bulk.

It must be mentioned that, although desirable and important, there has been no attempt so far to compare the hemispherical texturing with any other texturing scheme currently practiced and proposed. Also, for each particular case, a detailed full-cycle cost evaluation needs to be done. This first unambitious analysis might, notwithstanding its primitiveness, incite alternative ideas, based on the proposed technology, either for commodity or custom photovoltaic cells, and propel more in-depth research in this direction.

\section{Hemispherical Texturing Method}

The method employed to etch the array of smooth hemispherical depressions on the silicon surface is curiously based on bulk micromachining with an anisotropic etchant. The process is predictable, as follows, for a $\langle 100\rangle \mathrm{c}$-Si surface; $\mathrm{m}-\mathrm{Si}, \mu$-Si, $\mathrm{p}-\mathrm{Si}$ can also be deployed for such a texturing, where the extent of the added value being closely dependent on the density and size of $\langle 100\rangle$-oriented grains on the exposed surface. This texturing technique only requires a single oxide film with a mesh of openings and two etching steps in a $\mathrm{KOH}: \mathrm{H}_{2} \mathrm{O}$, or a TMAH: $\mathrm{H}_{2} \mathrm{O}$, solution. The use of a lithographic mask to create the opening mesh in $\mathrm{SiO}_{2}$ is not strictly necessary. Instead, at least two lower cost options can be deployed: (1) high-quality laser-printed translucent sheets or (2) poor porous sacrificial thermal oxide produced under nitrogen purge. There, the resulting mesh changes from a highly ordered to a random array of openings, both grid- and size-wise.

The texturing method is based on a seldom known subtlety of Si bulk etching: a $\langle 111\rangle$ pyramidal pit formed by a $\mathrm{KOH}$ anisotropic etch through an oxide mask eventually evolves into a smooth hemispherical cavity under subsequent maskless etching. An intermediate stage exists when a wider $19.47^{\circ}\langle 411\rangle$-faced pyramid replaces the initial $54.74^{\circ}$ inverted pyramid. The distance from base to vertex of the $\langle 411\rangle$-faced pyramid determines the depth of the resulting spherical cavity. The complete replacement of the $\langle 111\rangle$ walls by the $\langle 411\rangle$ ones occurs for an anterior wafer thinning $h_{\text {off }}$ of approximately $1 / 3$ of the $\langle 111\rangle$ initial pyramidal base dimension (i.e., $d_{0} / 3$ ). The base dimension $d_{0}$ is dictated by the largest dimension of the original opening on the oxide film. It is important to note that the initial film is not limited to $\mathrm{SiO}_{2}$ and can be actually any material that adheres well to $\mathrm{Si}$, is sufficiently resistant to the etchant used, and that can be easily and inexpensively removed later, as several polymers are. Figure 1 shows the evolution of the etching process after oxide removal.

Figure 2 indicates some useful parameters. The diameter $D$ of the hemispherical depression is determined by the size of the initial opening on the oxide mask $d_{o}$ and by the etch depth $h$, whereas its depth $s$ is determined by $d_{o}$ and the etching conditions. The empirical expression for the cavity diameter is $D=7.8 d_{0}^{0.42} h^{0.58}$, and it is valid for etch depths in excess of $2.5 d_{o}$ [9]; a perfectly round perimeter is obtained for $h>11 d_{o}$ [10]. The cavity depth, also referred to as sagitta, is $s=\alpha \cdot d_{o}$, where $\alpha$ is a process parameter that depends on the $\mathrm{KOH}$ concentration in the solution and on the temperature. At a thinning $h_{\text {off }}$ the wider $\langle 411\rangle$ pyramidal pit completely replaces the original $\langle 111\rangle$ walled one. Additional thinning $h^{*}$ is required to achieve a hemispherical shape.

The minimum initial opening is limited by the resolution of the exposure mask or by the minimum pore in the sacrificial oxide. Lithographic masks, laser-beam, ion-beam, or e-beam direct writing can define uniform sub-micrometric openings, whereas translucent sheets patterned by a printer define relatively uniform openings that are at least several microns large. Porous thermal $\mathrm{SiO}_{2}$, on the contrary, results in a random distribution of variously sized and shaped openings.

This method could be additionally combined with the traditional upward random-pyramids method (hillocks) to yield a yet more elaborate surface texture, as depicted in Figure 3, benefiting from the advantages of both.

\section{Light Transmittance}

Solar radiation can be decomposed into three components striking a ground-level photovoltaic cell: direct, diffuse, and albedo [11]. For the time being, nontilted cells and only the first radiation component are considered in our analysis, which is limited to the comparison between a surface textured with hemispherical cavities and a flat surface. Albedo radiation is mostly taken into account in tilted panels. Diffuse radiation can be as high as $15 \%$ of the impinging radiation, but due to its spatially random nature, it has been assumed to have equal contributions to transmittance on both surfaces considered here.

With respect to shading, the critical elevation angle $\gamma_{c}$ can be defined as that below which part of the impinging light is shaded by surface features. An orthogonal hemispherical cavity array is prone to increasing shading from a critical angle that depends on the process parameters, as in

$$
\gamma_{C}=\operatorname{tg}^{-1}\left(\frac{D}{2 s}\right)
$$




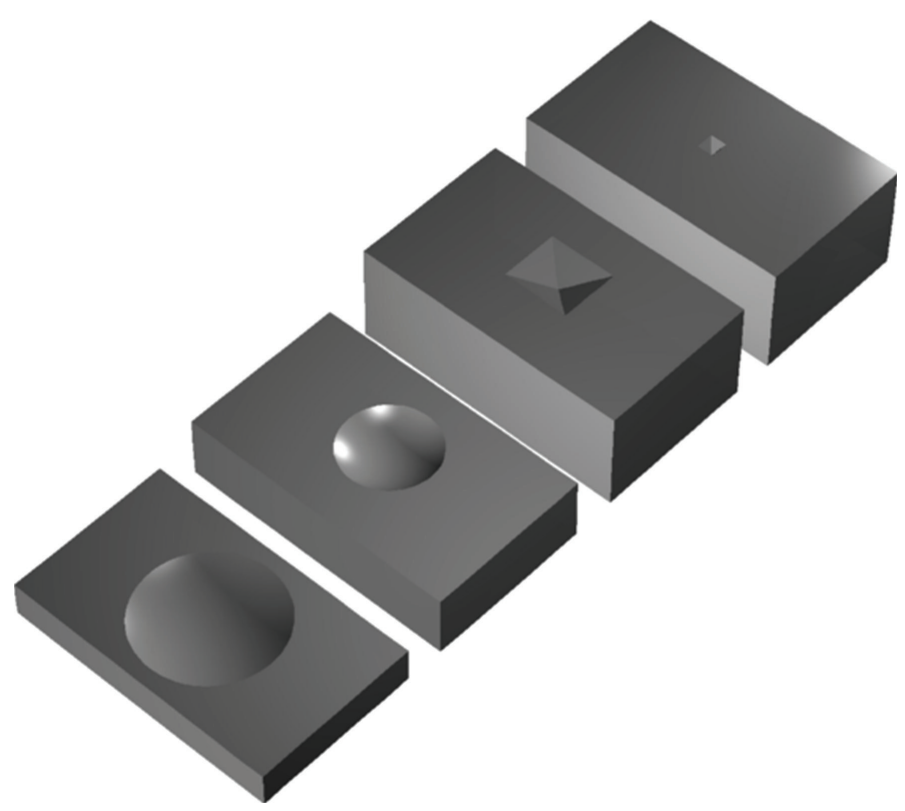

(a)
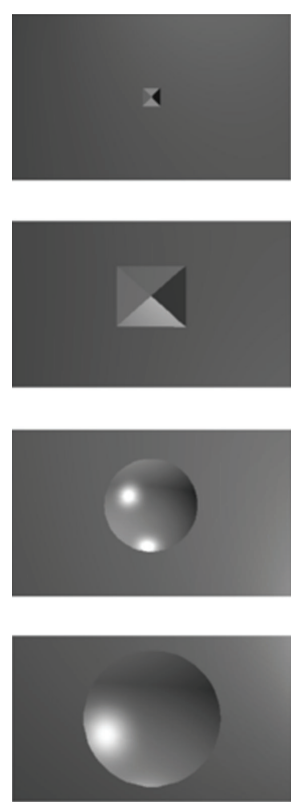

(b)

FIGURE 1: Evolution of an inverted pyramidal pit into a hemispherical depression. On the left one observes the concomitant thinning of the wafer. On the right one sees the cross-section and top view of the process.

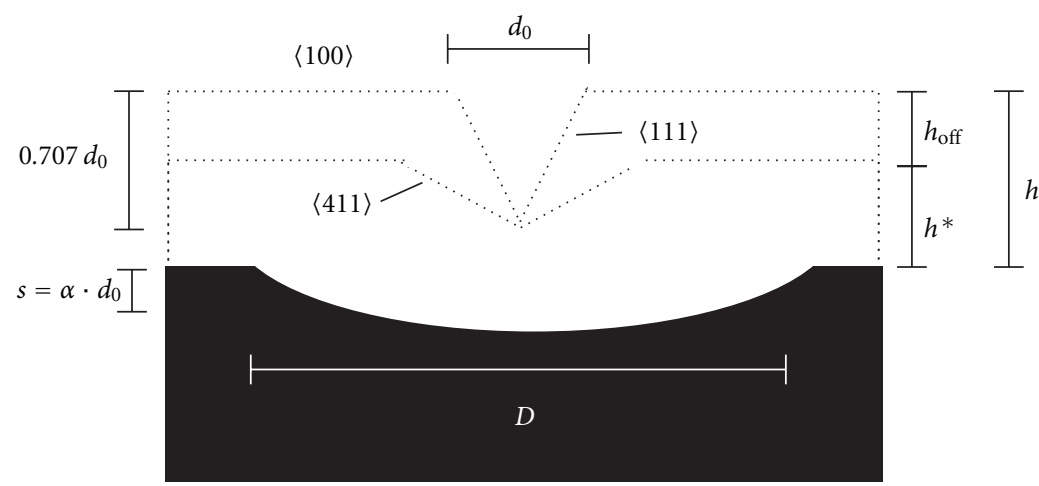

Figure 2: Parameters related to the etching process, from the initial pyramidal pit to the final hemispheric cavity.

or

$$
\gamma_{C}=\operatorname{tg}^{-1}\left[\frac{3.9}{\alpha}\left(\frac{h}{d_{o}}\right)^{0.58}\right] .
$$

Figure 4 illustrates the relationship between $\gamma_{c}$ and a single cavity.

The analysis that follows considers a 100\% fill-factor orthogonal array with sharp edges and sharp peaks at the grid intersection vertices, as is the case with this texturing technology. It does not include a sun-tracking system either. A surface with pyramidal pits, for instance, yields a critical angle $\gamma_{c}=54.74^{\circ}$, whereas the hemispherical approach results in critical angles from $2^{\circ}$ to $3^{\circ}$, when the ratio $h / d_{0}$ decreases from 5 to 2.5, as Figure 5 shows. Besides, in the hemispherical scheme, maximum shading is only significant when the sun trajectory is at $45^{\circ}$ with the matrix orientation, for which each intersection peak casts a shadow towards the center of its respective cavity.

The surface area of a truncated spherical cavity area is given by (3),

$$
\begin{aligned}
S_{\mathrm{cav}}= & 2 R\left[-2 R \cdot \operatorname{arctg}\left[\frac{P_{\max }^{2}}{4 R \sqrt{-\left(P_{\max }^{2} / 2\right)+R^{2}}}\right]\right. \\
& \left.+2 P_{\max } \cdot \operatorname{arctg}\left[\frac{P_{\max }}{2 \sqrt{-\left(P_{\max }^{2} / 2\right)+R^{2}}}\right]\right],
\end{aligned}
$$

where $R=D^{2} / 8 s$, and $P_{\max }$ is the maximum pitch between two cavity centers that still guarantees $100 \%$ structural fillfactor. For an orthogonal array, this parameter assumes a value $P_{\max }=D / \sqrt{2}$ and for a hexagonal array $P_{\max }=$ $1.5(D / \sqrt{3})$. Parameters $R$ and $P_{\max }$, ruling the cavity area, are dependent on design and process parameters $d_{0}$ and $h$, 

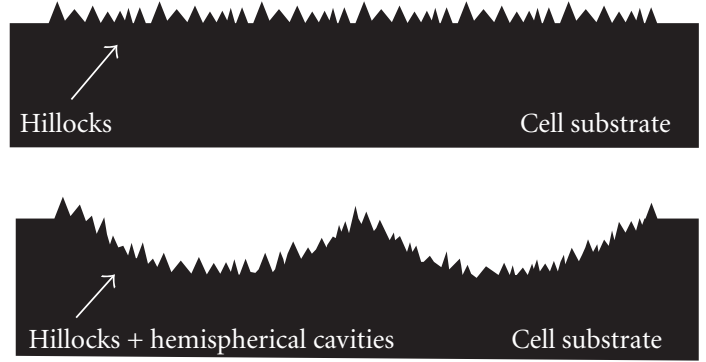

Figure 3: Schematic illustration of (top) front surface textured with the traditional random upward pyramids-hillocks; (bottom) front surface textured with a combination of hemispherical and random pyramids.

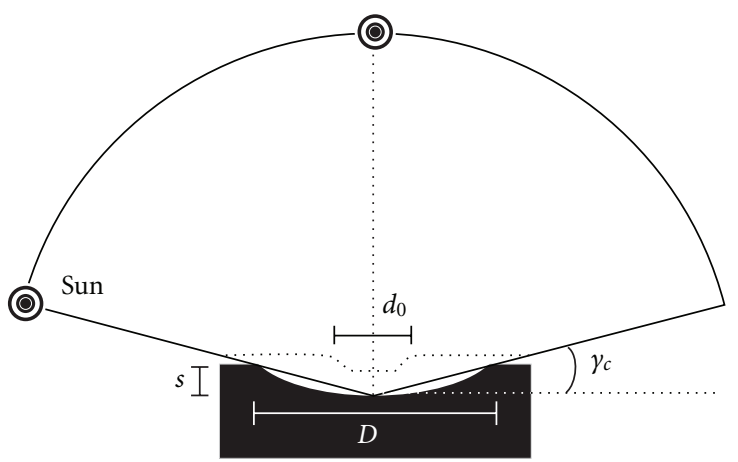

(a)
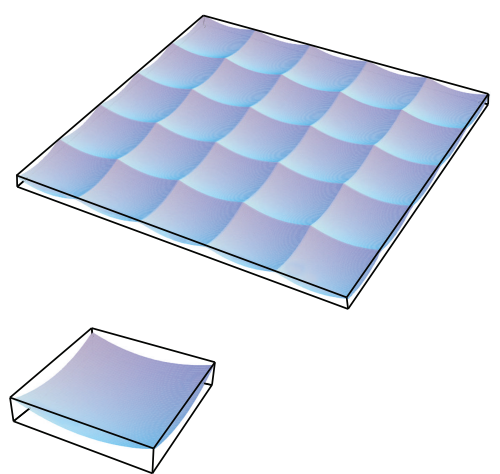

(b)

Figure 4: (a) Elevation angle and cell parameters for a single cavity, (b) orthogonal 100\% fill factor array, and a single truncated spherical cavity.

respectively. We have found that the area increases as the ratio $h / d_{0}$ decreases, resulting in an area gain of $0.35 \%$ with respect to a plane surface when $h / d_{0}=2.5$. The area gain is rather low; nevertheless, the effect of the hemispherical shape on the photon transmission should not be neglected.

We developed an algorithm to contrast the transmittance at the air-silicon interface of a flat cell to that of a hemispherically textured one. Sun rays sweep the cells from 0 to $90^{\circ}$ and the algorithm outputs the total normalized transmittance over the cell area. There is no sun-tracking mechanism. Figure 6 shows the graphs for the two cases,

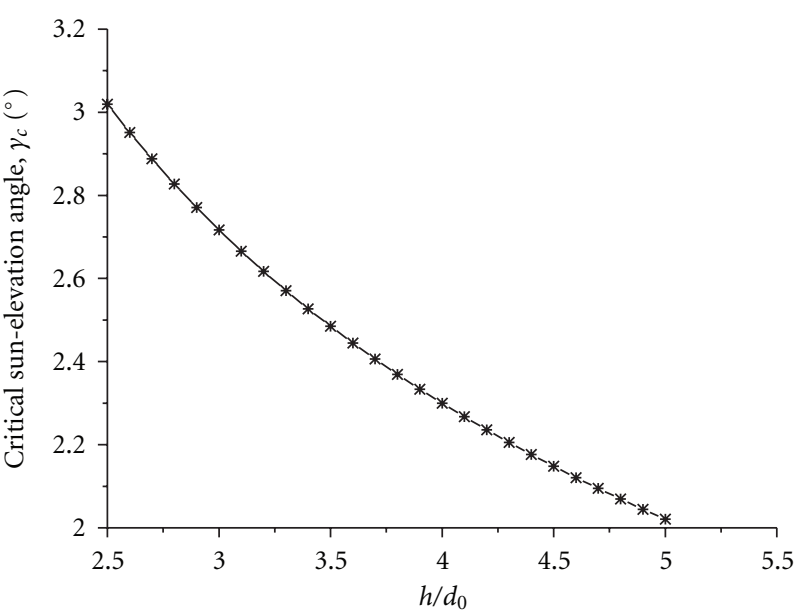

Figure 5: Critical sun-elevation angle $\gamma_{c}$ versus process ratio $h / d_{0}$. The angle $\gamma$ is measured from ground level and assumes $90^{\circ}$ at the Zenith.

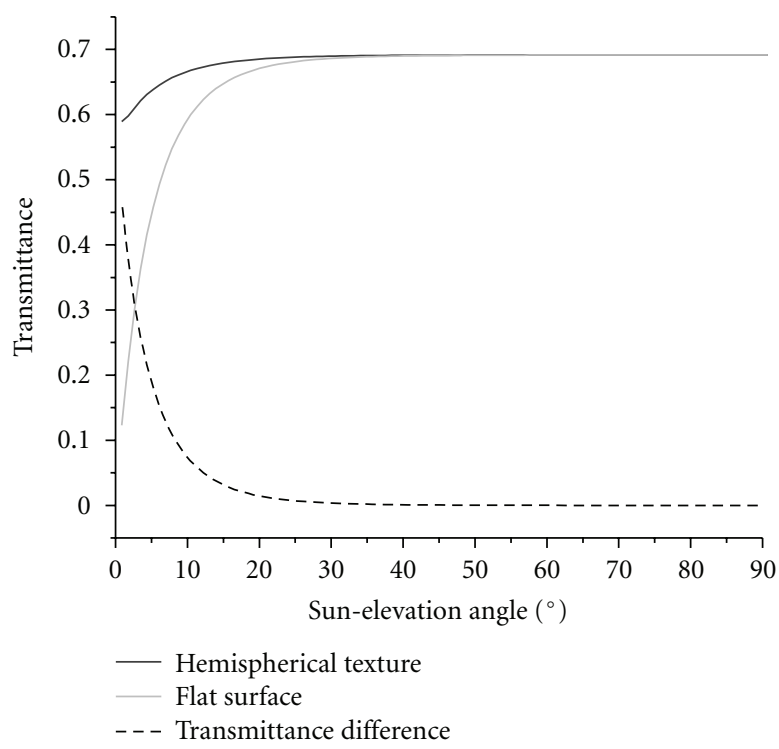

Figure 6: Transmittance as a function of sun-elevation angle $\gamma$ for nontilted flat and hemispherically textured cells; dashed: difference in transmittance.

where the textured surface has been obtained with the ratio $h / d_{0}=2.5$ and $d_{0}=40 \mu \mathrm{m}$.

These results show that the hemispherically textured cell exhibits considerable transmittance gain for elevation angles up to $30^{\circ}$. Using a textured cell, the transmittance can be larger than $30 \%$ higher for dawn and dusk hours. From geometrical optics, a factor contributing to this higher gain is the minimization of the highly oblique sun-ray angles with respect to surface loci, as more perpendicular incidence at each surface point yields a larger overall transmittance.

Additionally, we found out that the smaller the $h / d_{0}$ ratio and the larger the initial opening in the exposure mask $d_{0}$, the larger the transmittance. This leads to the conclusion that a large concave cell would perform the best, compared to a flat 


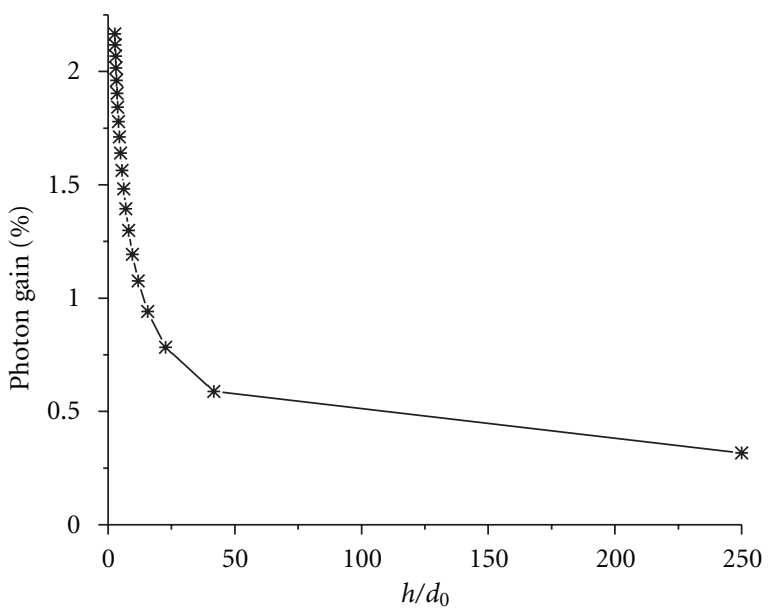

Figure 7: Gain in the number of absorbed photons for a hemispherically textured solar cell, as a function of the process ratio $h / d_{o}$, compared to a flat one in a nontilted condition, under the assumptions made in this section.

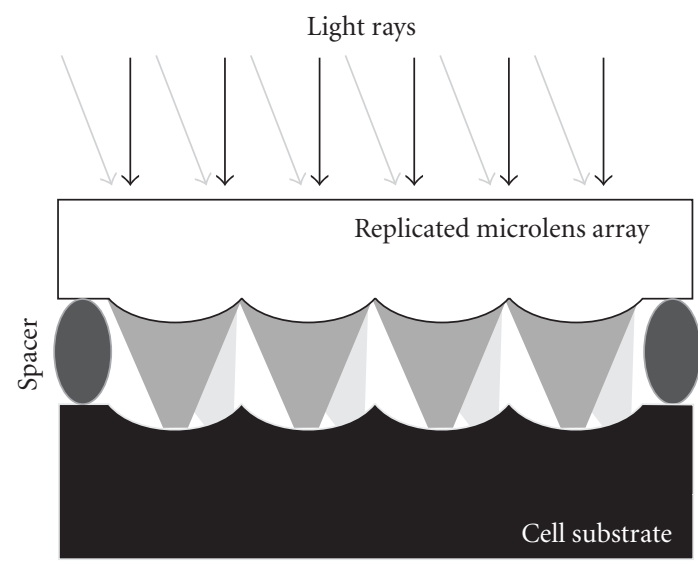

FIGURE 8: Microlens array replicated from the cell substrate itself. The microlens array is positioned at a distance from the substrate by means of spacers.

or a cavity-array surface. However, maximum $d_{0}$ is limited chiefly by the wafer thickness and the thinning $h=2.5 d_{0}$, which cares for the hemispherical shape. In practice this value ranges from $30 \mu \mathrm{m}$ to $160 \mu \mathrm{m}$ depending also on the back-end mechanical stability of the substrate. This range discards the need for high-resolution lithographic masks. For $100 \mathrm{~cm}^{2}$ surface the cost of a custom $\mathrm{Cr} / \mathrm{Ni}$-Quartz mask could be reduced from US $\$ 300$ to about US\$5 for highquality translucent sheets, and to no additional costs with the use of porous thermal oxide as a parasitic mask. Also, a single mask can be used for multiple lithographic exposures on larger surfaces.

To evaluate the benefits of a hemispherically textured cell compared to a flat cell, we calculated the total number of photons effectively penetrating each cell per day taking into account the previously calculated transmittance, and considering the average irradiance in Belo Horizonte, Minas Gerais (latitude $19^{\circ} 55^{\prime} \mathrm{S}$ and longitude $43^{\circ} 56^{\prime} \mathrm{W}$ ). We made a couple of simplifying assumptions with no detriment to our comparative analysis: the general characteristics of both cells are exactly the same, except for the anterior surface texturing; albedo and diffuse radiation are null; cells are at sea level and not tilted; sun-tracking system is absent; average wavelength $\bar{\lambda}=650 \mathrm{~nm}$ is taken as the center of gravity of the solar spectral distribution (ASTM-G173-03) from 280 to $1100 \mathrm{~nm}$ ( silicon cutoff wavelength); the sun is up $10 \mathrm{~h}$ per day.

Figure 7 shows the gain in number of photons as a function of $h / d_{0}$ for the conditions in the previous paragraph. We observe again that the lower $h / d_{0}$, the larger the gain. For $h / d_{0}=2.5$, the gain is higher than $2 \%$. Considering that a flat silicon surface absorbs an energy density of $\sim 900 \mathrm{kWh} / \mathrm{m}^{2}$ over a year [11], due to direct incidence at a location prone to medium irradiation, then a hemispherically textured cell can offer additional $18 \mathrm{kWh} / \mathrm{m}^{2}$ absorption. This value can range from $12 \mathrm{kWh} / \mathrm{m}^{2}$ to as high as $25 \mathrm{kWh} / \mathrm{m}^{2}$ for regions subjected to low and high irradiation levels, respectively.

The process parameter $h / d_{0}$ plays an important role in the design of an optimal hemispherical texture for the anterior surface of a solar cell. Although the critical shading angle increases as $h / d_{0}$ decreases, the area gain and transmittance gain are the largest for $h / d_{0}=2.5$. Also, the larger the mask opening size is, the largest the transmittance for low sunelevation angles. This favors the employment of low-cost printing of exposure masks for the texturing patterning, as opposed to high-definition lithographic masks.

The concave nature of the texturing acts as a highrefraction index diverging lens. This favors a longer propagation path of light inside the semiconductor, therefore increasing the absorption and photogenerated-carrier collection efficiency.

Also, the array of concave depressions can directly serve as a mould for a transparent UV-curable polymer or lowtemperature glass microlens array. The perfectly matched array of microlenses can be detached from the substrate and anchored at a small vertical distance from it by spacers. Sunlight through the convex lenses is refracted in such a way as to promote even less oblique incidence and concentrate light at the central region of each depression; also allowing space for a higher density of front-contact metallization, without compromising the effectively exposed area. The refractive microlens-array concentration is depicted in Figure 8.

\section{Substrate Fabrication}

We fabricated two textured $10 \mathrm{~mm} \times 10 \mathrm{~mm}$ substrate samples with hexagonal arrays of hemispherical cavities from starting circular openings with $d_{0}=3.4 \mu \mathrm{m}$ diameter and a pitch $P=300 \mu \mathrm{m}$. The substrate is a polished p-type (100) CZ monocrystalline silicon wafer. The oxide was a goodquality thermal wet oxide with $800 \mathrm{~nm}$ thickness.

The samples were etched in a $33 \mathrm{wt} \% \mathrm{KOH}: \mathrm{H}_{2} \mathrm{O}$ solution at $85^{\circ} \mathrm{C}$ and with no magnetic or ultrasound agitation. $\mathrm{SiO}_{2}$ was stripped with an HF solution. The absence of agitation and accurate temperature control are probably the reasons why there is some inhomogeneity in the cavity sizes 


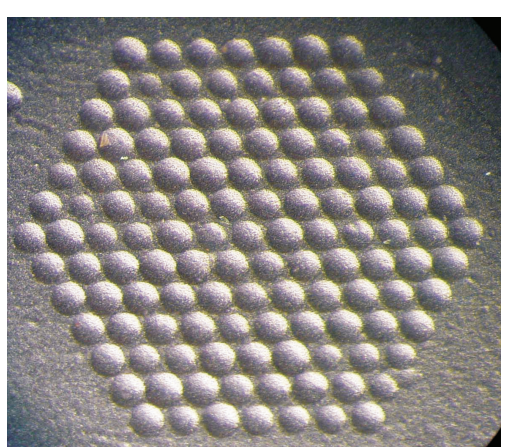

(a)

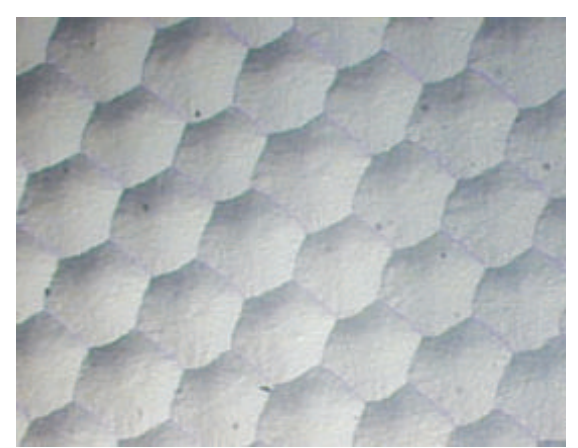

(b)

FIgURE 9: (a) Photograph of the array etched until most cavities touched each other edges; (b) photograph of the array etched until overlap of cavities resulted in $100 \%$ fill factor.

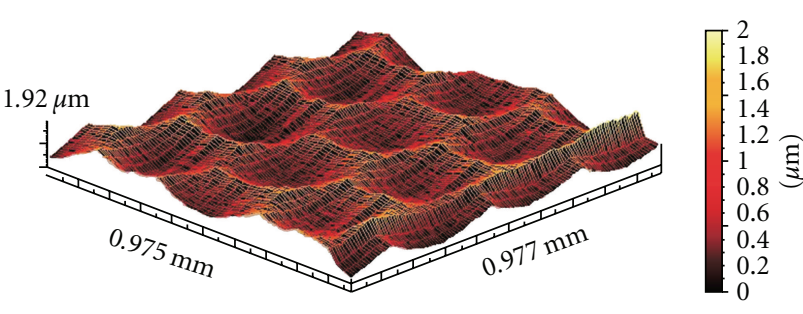

(a)

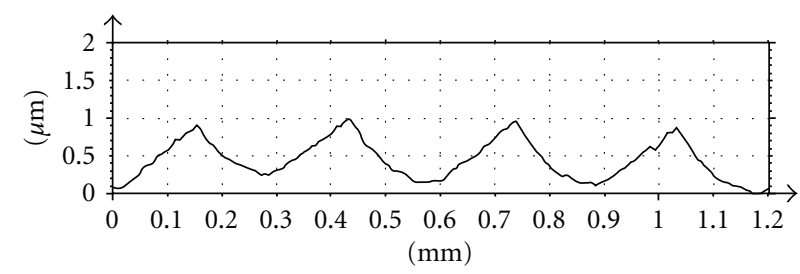

(b)

FIGURE 10: (a) 3D surface scanning; (b) linear scanning through the center of the sample.

and boundaries across the array, as the release of the etching by-products from the surface might not have been efficient. Results are shown in Figure 9.

Profilometry of the 100\%-fill-factor sample with a Taylor-Hobson Series 2 contact profilometer yielded the results shown in Figure 10. The average cavity depth, sagitta, is $0.83 \mu \mathrm{m}$.

We fabricated a subsequent $10 \mathrm{~mm} \times 10 \mathrm{~mm}$ sample with the same $\mathrm{KOH}$ solution concentration, but etched at $92^{\circ} \mathrm{C}$ $\pm 1^{\circ} \mathrm{C}$ with magnetic agitation of $590 \mathrm{rpm}$, and the sample suspended in the vertical position in $800 \mathrm{~mL}$ of the solution. Photolithography was realized with direct laser writer equipment (Microtech LW405). AFM analysis of this sample indicated a roughness of $4 \mathrm{~nm}$ on the (100) thinned surface and $1.8 \mathrm{~nm}$ around the center of the hemispherical cavity, prognosticating lower surface-recombination velocities.

\section{Discussion and Conclusions}

In this paper we showed that it is possible to etch a texture of (contiguous) hemispherical cavities in monocrystalline Si. Surprisingly, this can be achieved by anisotropic etching with smoothness as good as that from conventional chemomechanical polishing, so as to avoid deleteriously high surface recombination effects. The initial oxide openings preceding the texturing etch can be designed either with the high precision of lithographic masks and direct substrate writing, or with the medium precision of high-definition laser printers, or with the random distribution of porous thermal oxide. Also, this texturing technique could be combined with the traditional random-pyramid texture.

Given the shallow nature of the hemispherical cavities, calculations indicate a barely noticeable surface gain compared to a flat surface; however, the photon gain, mostly due to the minimization of oblique ray incidence, especially at dawn and dusk, is significant, resulting in an additional energy density from $12 \mathrm{kWh} / \mathrm{m}^{2}$ to as high as $25 \mathrm{kWh} / \mathrm{m}^{2}$ for regions subjected to low and high irradiation levels, under the assumptions specified in Section 3. The practical impact of these results, however, demands a comparison to both the performance and the processing cost of other textured surfaces already in use, which can be thoroughly tackled in a follow-up study.

Investigations need to be extended to include chargecarrier dynamics inside the semiconductor, but it can be already proposed that as the texture also acts as a concave lens, light will be refracted at angles further away from the vector normal to the surface, increasing the chances of absorption inside the material and closer to the emitter-base junction, but also enhancing the diffusion current-density component, influenced by both the diffusion length and the back-surface reflectance.

Another issue that deserves attention is the use of an anti-reflective coating. It is known that a $100 \mathrm{~nm}$ thick $\mathrm{SiO}_{2}$ film enhances by $10-30 \%$ the transmittance over a broad range of the Si-sensitive spectrum [12]. The presence of an anti-reflective coating does not invalidate any of the previous 
conclusions. On the contrary, it might even enhance these qualities, in addition to passivating surface states, and would benefit both planar and hemispherically textured surfaces.

Among the characteristics already discussed, the hemispherically textured substrate serves itself as a mould for the replication of polymeric or low-temperature glass microlens arrays, useful as local solar concentrators. Nevertheless, reflection and absorption by the microlens array also needs to be evaluated. If doping is performed after texturing, the space-charge region will present an increased volume, as compared to a flat emitter-base junction, and might render higher quantum efficiency, as already verified for photodiodes with wavy junctions [13].

Although the effective contribution of each single feature inherent to this technology can be arguable, the contribution of several features combined might prove useful.

\section{Acknowledgments}

The authors are thankful to Dr. Oleg Soloviev (OKO Technologies, NL), to Dr. Jeffrey Ketterling (Riverside Research Institute, USA), to Professor Dr. José A. Diniz (Unicamp), to Ms. Márcia Reutemberg (Unicamp), and to Dr. José Roberto T. Branco (CETEC-MG) for the useful discussions, and to FAPEMIG (Fundação de Amparo à Pesquisa do Estado de Minas Gerais, Brazil), CAPES, and CNPq (Conselho Nacional de Desenvolvimento Científico e Tecnológico, Brazil) for the financial support.

\section{References}

[1] D. H. Macdonald, A. Cuevas, M. J. Kerr et al., "Texturing industrial multicrystalline silicon solar cells," in Solar World Congress, Adelaide, Australia, 2001.

[2] A. J. Nijdam, E. Van Veenendaal, H. M. Cuppen et al., "Formation and stabilization of pyramidal etch hillocks on silicon 100 in anisotropic etchants: experiments and Monte Carlo simulation," Journal of Applied Physics, vol. 89, no. 7, pp. 4113-4123, 2001.

[3] P. Campbell, "Enhancement of light absorption from randomizing and geometric textures," Journal of the Optical Society of America B, vol. 10, no. 12, pp. 2410-2415, 1993.

[4] V. V. Iyengar, B. K. Nayak, and M. C. Gupta, "Optical properties of silicon light trapping structures for photovoltaics," Solar Energy Materials and Solar Cells, vol. 94, no. 12, pp. 22512257, 2010.

[5] H. Schröder, E. Obermeier, and A. Steckenborn, "Micropyramidal hillocks on $\mathrm{KOH}$ etched 100 silicon surfaces: formation, prevention and removal," Journal of Micromechanics and Microengineering, vol. 9, no. 2, pp. 139-145, 1999.

[6] F. Wang, H. Yu, J. Li et al., "Design guideline of high efficiency crystalline Si thin film solar cell with nanohole array textured surface," Journal of Applied Physics, vol. 109, no. 8, Article ID 084306, 5 pages, 2011.

[7] M. D. Kelzenberg, S. W. Boettcher, J. A. Petykiewicz et al., "Enhanced absorption and carrier collection in Si wire arrays for photovoltaic applications," Nature Materials, vol. 9, no. 3, pp. 239-244, 2010.

[8] D. L. Kendall, W. P. Eaton, and R. P. Manginell, "Micromirror arrays using $\mathrm{KOH}: \mathrm{H}_{2} \mathrm{O}$ micromachining of silicon for lens templates, geodesic lenses, and other applications," Optical Engineering, vol. 33, article 3578, 1994.

[9] D. W. de Lima Monteiro, O. Akhzar-Mehr, P. M. Sarro, and G. Vdovin, "Single-mask microfabrication of aspherical optics using $\mathrm{KOH}$ anisotropic etching of Si," Optics Express, vol. 11, no. 18, pp. 2244-2252, 2003.

[10] D. L. Kendall, G. R. de Guel, S. Guel-Sandoval, E. J. Garcia, and T. A. Allen, "Chemically etched micromirrors in silicon," Applied Physics Letters, vol. 52, no. 10, pp. 836-837, 1988.

[11] J. Nelson, The Physics of Solar Cells, Imperial College Press, 2003.

[12] W. J. Kindt, Geiger mode avalanche photodiode array, Ph.D. dissertation, Faculty of Information Technology and Systems, Delft University Press, Delft, The Netherlands, 1999.

[13] T. Coura, L. P. Salles, and D. W. de Lima Monteiro, "Quantumefficiency enhancement of CMOS photodiodes by deliberate violation of design rules," Sensors and Actuators, A, vol. 171, no. 2, pp. 109-117, 2011. 


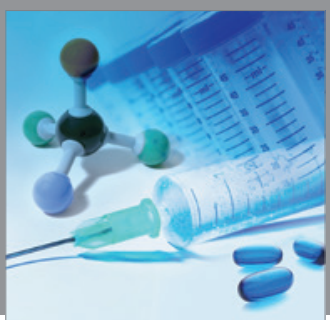

International Journal of

Medicinal Chemistry

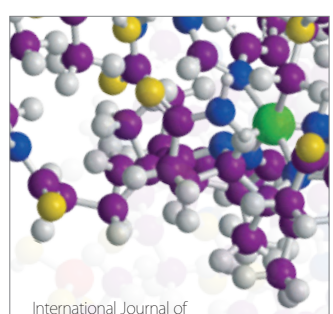

Carbohydrate Chemistry

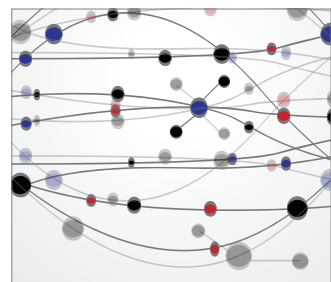

The Scientific World Journal
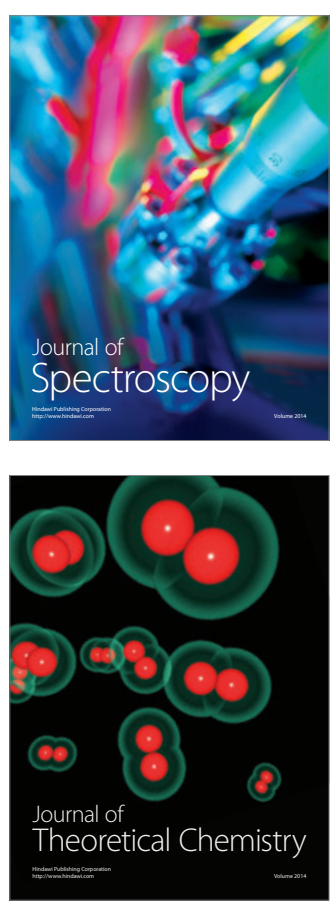
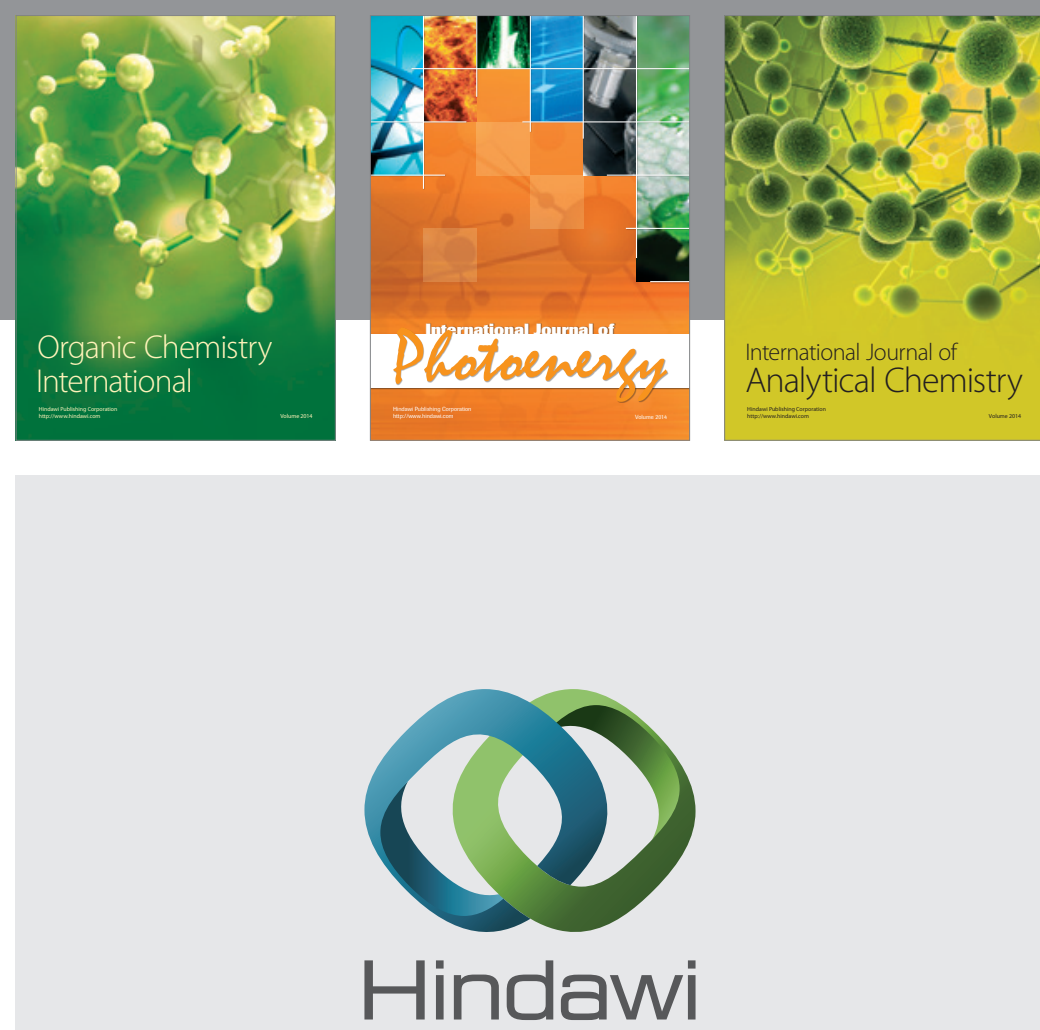

Submit your manuscripts at

http://www.hindawi.com
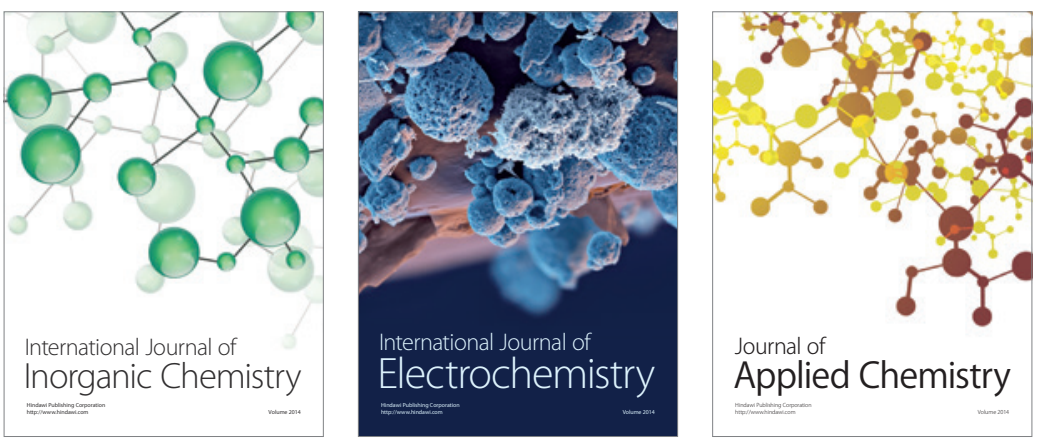

Journal of

Applied Chemistry
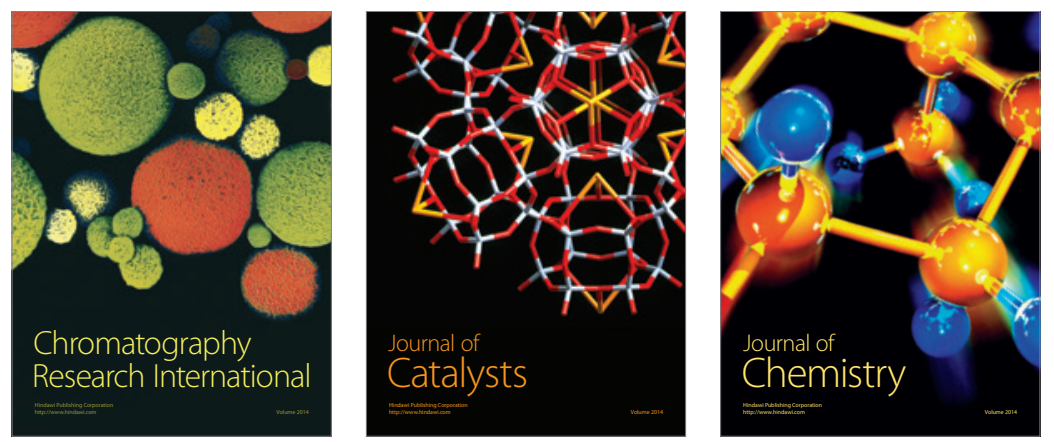
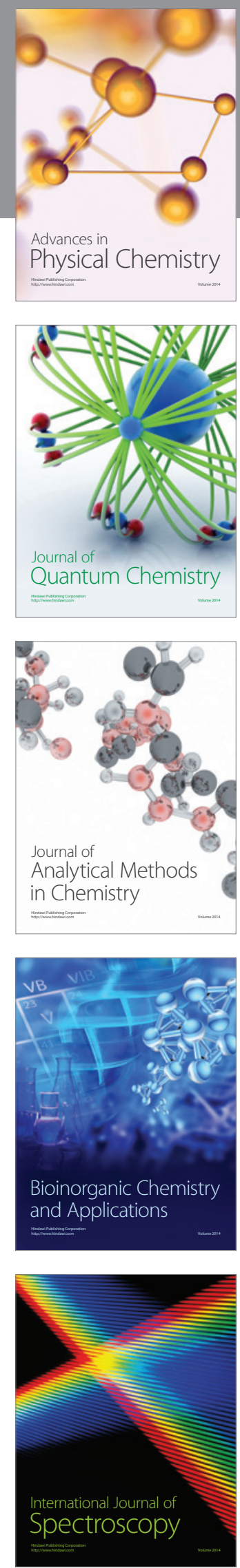